Graduate Students

\title{
OPTIMIZATION MODELS FOR MULTIPLE RESOURCE PLANNING
}

Norah Mohammed Z Al-dossaria ${ }^{a}$ Mohamed Kharbeche ${ }^{b}$, Mohamed Haouaria

a Mechanical and Industrial Engineering Department, ${ }^{b}$ Qatar Transportation and Traffic Safety Center, Qatar University

\section{Abstract}

- Multiple Resource Planning (MRP) is a very crucial undertaking for most organizations.

- MRLPs are very prevalent in today's organizational environments and are particularly critical for organizations that handle concurrent, timeintensive, and multiple-resource projects.

- MRP facilitates efficient allocation of resources and reduces costs.

- Using data obtained from the Ministry of Administrative Development, Labour and Social Affairs (ADLSA), an MRLP is proposed.

- A novel models and solution approach for the MRLP were proposed.

- The results show that the model performs well, even in higher instances.

- The positive results attest to the effectiveness of the proposed MRLP problem.

\section{Introduction/Objectives}

\begin{tabular}{|c|c|c|}
\hline $\begin{array}{l}\text { Create a solution that } \\
\text { solves the key } \\
\text { challenges r that } \\
\text { project managers } \\
\text { encounter in day-to- } \\
\text { day management of } \\
\text { multiple projects. }\end{array}$ & $\begin{array}{l}\text { Dealing with high } \\
\text { project demands, } \\
\text { uncertainties, project } \\
\text { constraints, and } \\
\text { dealing } \\
\text { competing priorities. }\end{array}$ & $\begin{array}{l}\text { Enhance project } \\
\text { scheduling within a } \\
\text { given time horizon } \\
\text { to get the optimal } \\
\text { resource allocation } \\
\text { solution. }\end{array}$ \\
\hline
\end{tabular}

\section{Methods and Materials}

\section{* Input Data:}

$\boldsymbol{n}$ : Number of tasks,

$\boldsymbol{R}:$ Number of resources,

$\boldsymbol{H}$ : Time horizon,

$\boldsymbol{b}_{r t}$ : Capacity of resource $r$ at period $t$,

$\boldsymbol{m}_{i}$ : Number of execution modes of task $j$,

$\boldsymbol{a}_{j r k}$ : Consumption of resource $r$ by task $j$ under mode $k$,

$\boldsymbol{p}_{j}$ : Processing time of task $j$ under mode $k$,

$\boldsymbol{r}_{j}$ : start date of the project

$\boldsymbol{d}_{j}$ : Due date of task $j$,

$\boldsymbol{w}_{j}$ : Weight of task $j$,

$\sigma_{r t}$ : Cost of adding one unit of capacity to resource $r$ at period $t$.

\section{* Decision Variables:}

$\boldsymbol{x}_{\boldsymbol{j} \mathbf{k}}$ : Binary variable that takes value 1 if task $j$ is executed under mode $k$ and 0 otherwise.

$y_{j t}$ : Binary variable that takes value 1 if task $j$ is executed during period $t$, and 0 otherwise.

$\boldsymbol{s}_{j t}$ : Binary variainle that takes value 1 if task $j$ starts at the beginning of period $t$, and 0 otherwise (that means, $s_{j t}=1 \Rightarrow$ task $j$ starts at time $t$ ).

$\boldsymbol{f}_{j t}$ : Binary variable that takes value 1 if task $j$ finishes at the end of period $t$, and 0 otherwise (that means, $\mathrm{f}_{\mathrm{jt}}=1 \Rightarrow$ task $j$ finishes at time $t+1$ ).

$T_{j}$ : Tardiness of task $j$.

$\boldsymbol{z}_{\boldsymbol{r t}}$ : Additional capacity of resource $r$ at period $t$

\section{*Test Instances Ranges:}

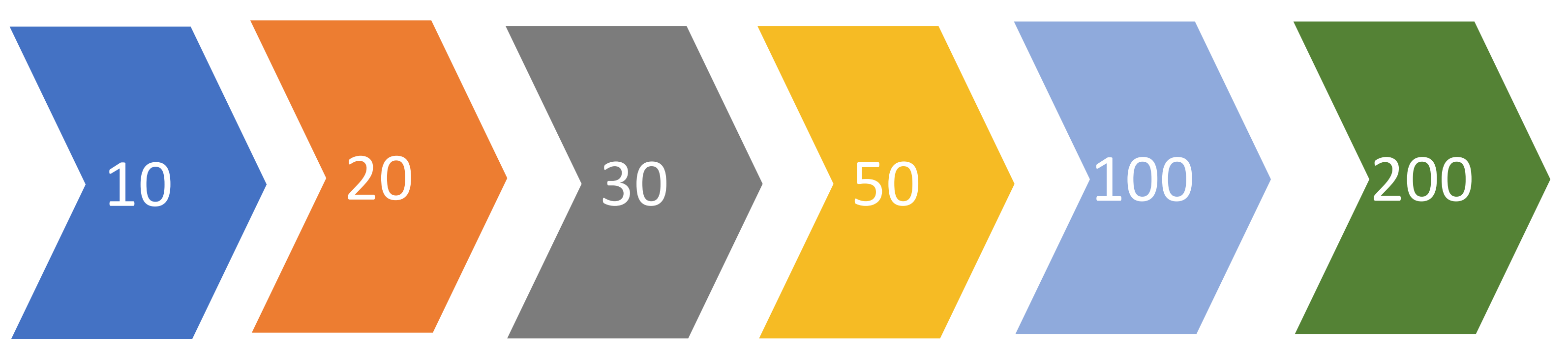

Figure 1. Number of projects ranges

\section{Model Formulation}

Model (MM, MR, FC): Minimize $\sum_{j=1}^{n} w_{j} T_{j}+\sum_{r=1}^{R} \sum_{t=1}^{H} \sigma_{r t} z_{r t}$

Subject to:
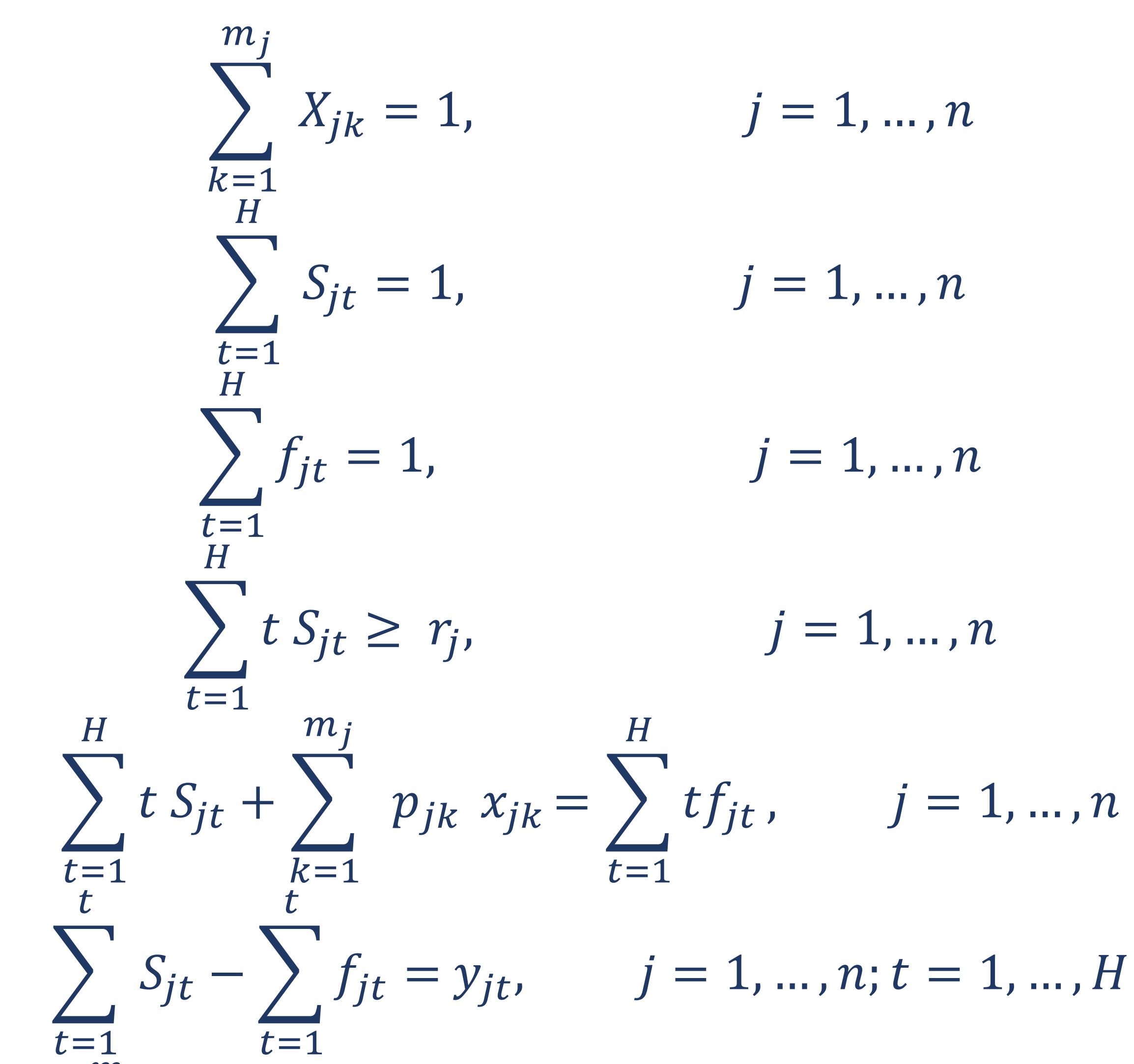$$
\sum_{j=1}^{n} \sum_{k=1}^{m_{j}} a_{j r k} u_{j k t} \leq b_{r t}+z_{r t} \quad r=1, \ldots, R, ; t=1, \ldots, H
$$$$
T_{j} \geq \sum_{t=1}^{H} t f_{j t}-d_{j}, \quad j=1, \ldots, n
$$$$
x_{j k}+y_{j t} \leq u_{j k t}+1, \quad j=1, \ldots, n ; R=1, \ldots, m_{j} ; t=1, \ldots, H
$$

$T, s, f, u \geq 0$,

\section{Results \& Discussion}

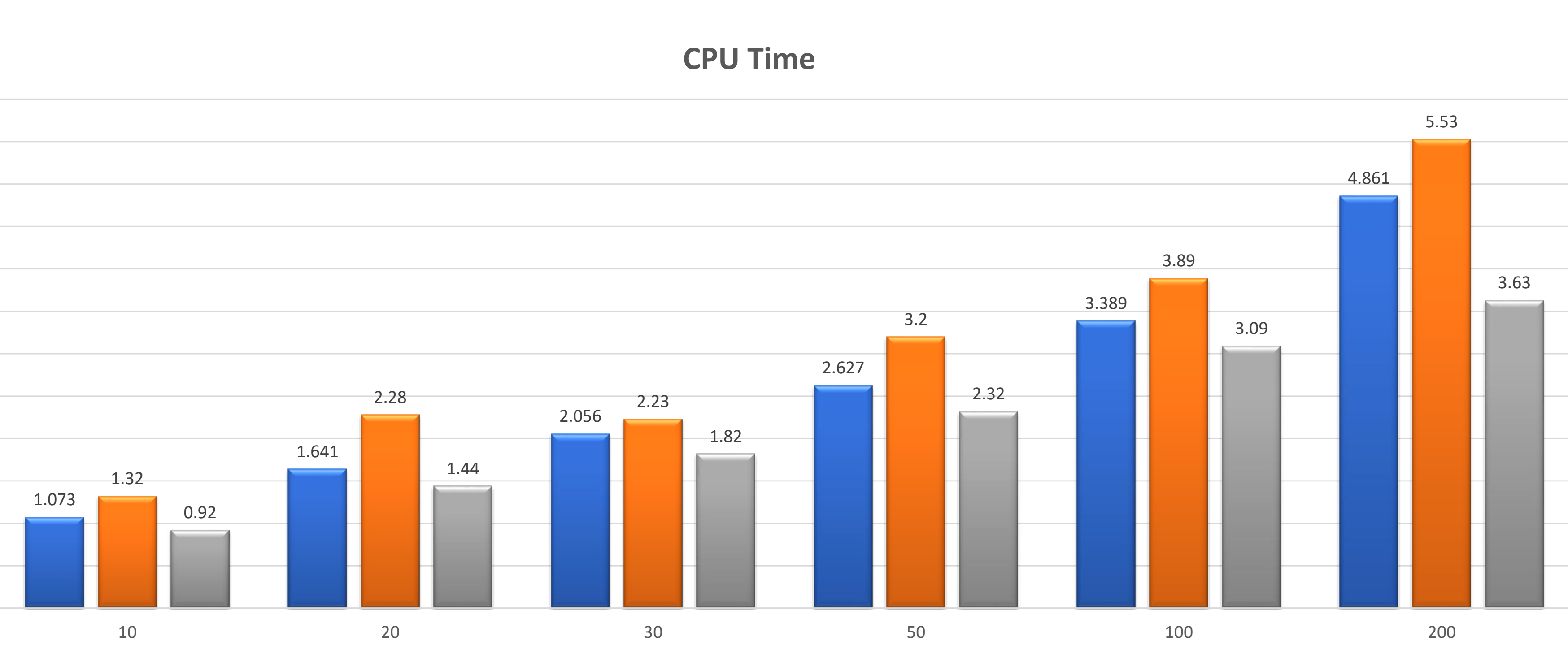

Figure 2. CPU time of the model

As the complexity of the test instances increased, the CPU time has increased reasonably. The proposed model was performed within a very short time (seconds) even when increasing complexity of tasks number to 200.

- The maximum running time was completed in 5.53 seconds while the average time-solving time is between 1.073 and 4.861 seconds.

\section{Conclusions}

Provides a solution that minimizes time wastages and ensures efficient resource utilization for both single-mode and multi modes projects, while allows the combination of resources while considering restrictions and time constraints to achieve optimal scheduling and resource planning.

References

Bianco, L., \& Caramia, M. (2013). A new formulation for the project scheduling problem under limited resources. Flexible Services and Manufacturing Journal, 25(1-2), 6-24. Chakrabortty, R., Abbasi, A., \& Ryan, M. (2019). Multi-mode resource-constrained project scheduling using modified variable neighborhood search heuristic. International

\section{Contact}

NORAH MOHAMMED Z AL-DOSSARI

Mechanical and Industrial Engineering Department 